\title{
The Role of Inflammatory Factors in the Covid-19 Disease
}

Fesharaki MJ ${ }^{1}$, Eslami V1, Sandgol G², Gharaei $\mathrm{B}^{3}$, Vahidi $\mathrm{M}^{4}$, Rezaei $\mathrm{B}^{4}$, Abdi $\mathrm{S}^{5}$, Pirsalehi $\mathrm{A}^{6}$ and Shirini $\mathbf{D}^{2 *}$

${ }^{1}$ Assistant Professor of Cardiology, Cardiovascular Research Center, Shahid Beheshti University of Medical Sciences, Tehran, Iran

${ }^{2}$ Cardiovascular Research Center, Shahid Beheshti

University of Medical Sciences, Tehran, Iran

${ }^{3}$ Department of Anesthesia, Medical School, Shahid

Beheshti University, Tehran, Iran

${ }^{4}$ Faculty of Medicine, Shahid Beheshti University of Medical Sciences, Tehran, Iran

${ }^{5}$ Gastroenterology and Liver Diseases Research Center, Shahid Beheshti University of Medical Sciences, Tehran, Iran

${ }^{6}$ Taleghani Hospital Research Development Committee, Medical School, Shahid Beheshti University of Medical Sciences, Tehran, Iran

*Corresponding author: Dorsa Shirini, Cardiovascular Research Center, Shahid Beheshti University of Medical Sciences, Tehran, Iran

Received: May 26, 2021; Accepted: July 10, 2021; Published: July 17, 2021

\section{Abstract}

Late in 2019 a new pandemic was caused by a novel coronavirus which was later named as Covid-19 by WHO. Regarding the unknown behavior of the disease finding some predicting factors was important so it could be possible to curb the mortality and morbidity risk of the disease. Later studies showed the important role of inflammatory factors in the process of the disease. In this retrospective, multicenter study, 1199 adult patients were randomly selected. Patients were divided to three main groups: Severe/Moderate, ICU add/NonICU add and Expired/Alive. The clinical data (including heart rate, respiratory rate, systolic blood pressure and diastolic blood pressure) and the laboratory findings (including NLR, PLR, D-dimer, Troponin, and CRP) of their first date of admission were studied from their documents. The mean of each factor was compared within each group and the binary logistic regression was done for each factor. ROC curves were drawn separately based on the results of the binary logistic regression analysis $(P$-value $<0.05)$. Out of 1199 patients, 908 were in the severe group, 143 were ICU added and 144 were expired. Age and clinical factors were significantly higher $(P$-value $=0.000)$ in the severe, ICU add, and expired groups. All the inflammatory factors were significantly higher in the severe group $(P$-value $=0.000)$, were higher among ICU add patients with statistical significance of CRP, NLR, and PLR (P-value $=0.000,0.000$ and 0.001 respectively), and were higher in the expired group with the statistically significant difference in CRP, Troponin, NLR, and PLR ( $P$-value $=0.000)$.

Keywords: Covid-19; Inflammatory factors; CRP; D-dimer; NLR; PLR; Troponin

\section{Abbreviations}

WHO: World Health Organization; ICU: Intensive Care Unit; NLR: Neutrophil to Lymphocyte Ratio; PLR: Platelet to Lymphocyte Ratio; CRP: C-Reactive Protein; ROC Curve: Receiver Operating Characteristic Curve; SARS-CoV-2: Severe acute respiratory syndrome coronavirus 2; CT Scan: Computerized Tomography Scan; NSAID: Non-steroidal anti-inflammatory drugs; RR: Respiratory Rate; HR: Heart Rate; SBP: Systolic Blood Pressure; DBP: Diastolic Blood Pressure; IL-6: Interleukin-6

\section{Introduction}

The disease which was caused by a novel coronavirus, named as SARS-CoV-2, in 2019 (Covid-19) has affected millions of people around the world as a pandemic [1]. At first this virus was detected in February 2020 in Wuhan, China [2,3]. In comparison with seasonal influenza, it has higher virulence rate and can cause more hospitalizations and deaths $[4,5]$. The disease has a wide range of clinical presentations from no symptoms to acute respiratory and even worse, multi-organ failure and death [4]. Patients with risk factors such as older age or comorbidities such as diabetes or chronic respiratory disease are more vulnerable than those without risk factors [6]. In addition, patients with critical symptoms are more likely to get admitted to intensive care units, have longer hospitalization periods and die in comparison with those who have mild to moderate symptoms [7].

Inflammation and following severe inflammatory responses that can cause immune response imbalance, which can lead to viral hyper inflammation, play an important role in progression of Covid-19 [8-10]. Many studies showed that significant changes in different hematologic parameters such as lymphocytes, neutrophils, platelets, C-reactive protein (CRP), D-Dimer and cardiac troponin $[11,12]$ can lead to critical health problems. Also, there are strong evidences showing Neutrophil to Lymphocyte Ratio (NLR) and Platelet to Lymphocyte Ratio (PLR) both have prognostic value in the severity of the disease [13-17]. Moreover, there was significant increase in D-dimer, CRP, and c-Troponin in severe cases $[12,18]$. Therefore, it will be useful, especially in patients with mild to moderate symptoms at the beginning, to study these factors in order to early intervention to prevent severe outcomes.

Despite the importance of these factors, most of the researches are still focused on epidemiology and clinical presentations of Covid-19 [2]. As a result, our knowledge about the inflammation and immune response of this disease is still elusive. In this research we wish to investigate the effect of all of these factors more detailed and the probability of their use as prognostic factors of the severity of the disease.

\section{Methods}

\section{Patient selection}

We performed a retrospective, observational and multi-central study. For this purpose, 1199 adult ( $\geq 18$ years) patients were selected randomly admitted from February 20 to April 20, 2020, characterized by their symptoms, based on the guidance of the CDC and WHO [19], and compatible chest CT findings. Patients with RR $\geq 30$ times/ 
min and oxygen saturation (Resting state) $\leq 90 \%$ were known as severe group and those who didn't meet the criteria were defined as moderate group (292 severe and 917 moderate cases). All these selected patients were treated by regimes suggested by national guidelines.

\section{Data collection}

We studied electronic medical records including demographic findings, past medical and past drug history, signs and symptoms, medical treatment regimens, laboratory findings, ECGs and imaging findings (lung CT-scan). Laboratory findings (CBC: Lymphocyte, Neutrophil, Platelet, CRP, Troponin, D-Dimer) all were collected in organized sheets. All of the lab findings were registered at the admission date. Patients with the history of inflammatory diseases or usage of drugs such as glucocorticoids or NSAIDS were excluded from the study (as these factors can change the inflammatory profile).

\section{Statistical analysis}

At the first step the data was documented in the forms that were written by two medical students who were well-educated in Covid-19 in excel software. Then the data was exported to SPSS (IBM SPSS statistics version 26) and the analyses that have been applied to the data were: one sample T-test, binary logistic regression, and at last the ROC curve was drawn based on the results of previous tests. Histograms, measures of skewness and kurtosis, and KolmogorovSmirnov test were used to show the normality of the distribution of continuous factors (mean \pm SD and the range between $25-75 \%$ respectively). The relationship of NLR, PLR, CRP, Troponin, and $\mathrm{D}$-dimer as continuous variables with the severity, mortality risk and the risk of ICU admission were also studied. The clinical data including age, Respiratory Rate (RR), Heart Rate (HR), Systolic Blood Pressure (SBP), and the Diastolic Blood Pressure (DBP) were analyzed by sample T-test. The results were considered statistically significant with $\mathrm{P}$-value $<0.05$.

\section{Results}

In this retrospective study, 1199 adult patients ( $\geq 18$ years old) were randomly selected, including 735 men and 464 women. The mean age was 52.21 (18-102) with a maximum of 102. Out of these patients, 908 (who met the criteria of WHO) were in the severe group and 291 were in the moderate group, 143 were ICU added and 144 were expired. Laboratory factors including NLR $(\mathrm{N}=886)$, PLR ( $\mathrm{N}=900), \mathrm{CRP}(\mathrm{N}=820)$, Troponin $(\mathrm{N}=470)$, and $\mathrm{D}$-dimer $(\mathrm{N}=63)$ on the admission date were studied within three different categories: a) Severe or Moderate; b) ICU add or Non-ICU add and c) Expired or Alive. Age and some clinical factors (RR, HR, SBP, and DBP) were also studied within these three groups. Age and clinical factors were significantly higher $(\mathrm{P}$-value $=0.000)$ in the severe, ICU add patients, and expired groups in comparison to moderate, Non-ICU add, and alive ones, respectively (Table 1). In comparison between severe and moderate groups (Table 2) all the inflammatory factors that had been investigated in this study, were significantly higher in the severe group (P-value=0.000). In ICU add/Non-ICU add; all the laboratory parameters were higher among patients who were admitted to the Intensive Care Unit (ICU) (Table 3). These differences, between ICU add and Non-ICU add group, were statistically significant about CRP, NLR, and PLR (P-value $=0.000,0.000$ and 0.001, respectively). Despite being higher, Troponin and D-dimer had no statistically significant difference between these two groups $(\mathrm{P}$-value $=0.390$, 0.115 , respectively). In the case of expired /alive groups, all of the laboratory findings were higher in the expired group than alive ones (Table 4). The difference in CRP, Troponin, NLR, and PLR on admission date between these two groups was statistically significant $(\mathrm{P}$-value $=0.000)$. Although $\mathrm{D}$-dimer was higher in the expired group, this difference was not significant $(\mathrm{P}$-value $=0.631)$.

In each category, statistically significant laboratory parameters (P-value $<0.05)$ were included in binary logistic regression. Within the severe/moderate groups all of the laboratory factors were independent risk factors for the severity of the disease (Table 4). Within the ICU add/non-ICU add groups NLR, PLR, and CRP were independent risk factors for the admission in the intensive care unit (ICU) (Table 5). And in the third category, within expired/alive groups all the laboratory factors that have been investigated in this

Table 1: Clinical Factors.

\begin{tabular}{|c|c|c|c|c|c|c|c|c|c|c|}
\hline & RR Mean \pm SD & $P$ value & SBP Mean \pm SD & P Value & DBP Mean \pm SD & $P$ value & HR Mean \pm SD & $P$ value & Age Mean \pm SD & $P$ value \\
\hline Severe & $\begin{array}{c}20.10 \pm 5.82 \\
(N=266)\end{array}$ & 0.000 & $\begin{array}{c}121.95 \pm 21.93 \\
(\mathrm{~N}=284)\end{array}$ & 0.000 & $\begin{array}{c}75.74 \pm 12.72 \\
(\mathrm{~N}=282)\end{array}$ & 0.000 & $\begin{array}{c}91.57 \pm 17.40 \\
(\mathrm{~N}=284)\end{array}$ & 0.000 & $\begin{array}{c}65.26 \pm 16.32 \\
(N=291)\end{array}$ & 0.000 \\
\hline Moderate & $\begin{array}{c}18.17 \pm 5.28 \\
(\mathrm{~N}=858)\end{array}$ & & $\begin{array}{c}117.65 \pm 15.11 \\
(\mathrm{~N}=885)\end{array}$ & & $\begin{array}{c}74.67 \pm 9.69 \\
(\mathrm{~N}=882)\end{array}$ & & $\begin{array}{c}87.17 \pm 14.22 \\
(\mathrm{~N}=890)\end{array}$ & & $\begin{array}{c}48.04 \pm 17.82 \\
(\mathrm{~N}=908)\end{array}$ & \\
\hline ICU add & $\begin{array}{c}20.29 \pm 6.21 \\
(N=127)\end{array}$ & 0.000 & $\begin{array}{c}124.79 \pm 27.30 \\
(N=143)\end{array}$ & 0.000 & $\begin{array}{c}75.97 \pm 15.65 \\
(\mathrm{~N}=144)\end{array}$ & 0.000 & $\begin{array}{c}92.39 \pm 20.46 \\
(N=142)\end{array}$ & 0.000 & $66.31 \pm 17.01(143)$ & 0.000 \\
\hline $\begin{array}{l}\text { Non-ICU } \\
\text { add }\end{array}$ & $18.42 \pm 5.34(997)$ & & $\begin{array}{c}117.84 \pm 14.98 \\
(N=1026)\end{array}$ & & $\begin{array}{c}74.72 \pm 9.73 \\
(\mathrm{~N}=1020)\end{array}$ & & $\begin{array}{c}87.66 \pm 14.20 \\
(1032)\end{array}$ & & $50.31 \pm 18.04(156)$ & \\
\hline Expired & $\begin{array}{c}21.87 \pm 5.73 \\
(N=123)\end{array}$ & 0.000 & $\begin{array}{c}122.70 \pm 27.45 \\
(\mathrm{~N}=139)\end{array}$ & 0.000 & $\begin{array}{c}75.32 \pm 16.47 \\
(\mathrm{~N}=123)\end{array}$ & 0.000 & $\begin{array}{c}92.56 \pm 22.12 \\
(N=138)\end{array}$ & 0.000 & $\begin{array}{c}72.24 \pm 14.06 \\
(N=144)\end{array}$ & 0.000 \\
\hline Alive & $\begin{array}{c}18.23 \pm 5.03 \\
(N=1001)\end{array}$ & & $\begin{array}{c}118.15 \pm 15.13 \\
(N=1030)\end{array}$ & & $\begin{array}{c}74.82 \pm 9.63 \\
(N=1029)\end{array}$ & & $\begin{array}{c}87.66 \pm 13.89 \\
(N=1036)\end{array}$ & & $\begin{array}{c}49.49 \pm 17.87 \\
(N=1055)\end{array}$ & \\
\hline
\end{tabular}

Table 2: Severe/Moderate

\begin{tabular}{|c|c|c|c|c|}
\hline & Total Mean & Severe Group (Mean \pm SD) & Moderate Group (Mean \pm SD) & P-Value \\
\hline CRP & $35.73(\mathrm{~N}=820)$ & $51.81 \pm 43.52(\mathrm{~N}=233)$ & $29.30 \pm 31.46(\mathrm{~N}=587)$ & 0.000 \\
\hline Troponin & $0.63(\mathrm{~N}=470)$ & $1.49 \pm 7.62(\mathrm{~N}=158)$ & $0.20 \pm 0.43(\mathrm{~N}=312)$ & 0.000 \\
\hline D-dimer & $3233.48(\mathrm{~N}=63)$ & $5290.03 \pm 6395.81(\mathrm{~N}=29)$ & $1479.36 \pm 3080.69(\mathrm{~N}=34)$ & 0.000 \\
\hline NLR (Neutrophil to Lymphocyte Ratio) & $5.11(\mathrm{~N}=886)$ & $6.61 \pm 4.60(\mathrm{~N}=253)$ & $4.51 \pm 4.59(\mathrm{~N}=633)$ & 0.000 \\
\hline PLR (Platelet to Lymphocyte Ratio) & $13.03(\mathrm{~N}=900)$ & $15.33 \pm 12.65(\mathrm{~N}=255)$ & $12.12 \pm 17.53(\mathrm{~N}=645)$ & 0.008 \\
\hline
\end{tabular}


Table 3: ICU-add/Non-ICU-add.

\begin{tabular}{|c|c|c|c|c|}
\hline & Total Mean & ICU-add Group (Mean \pm SD) & Non-ICU-add Group (Mean \pm SD) & P-Value \\
\hline CRP & $35.73(\mathrm{~N}=820)$ & $55.03 \pm 50.36(\mathrm{~N}=130)$ & $32.05 \pm 32.31(\mathrm{~N}=690)$ & 0.000 \\
\hline Troponin & $0.63(\mathrm{~N}=470)$ & $0.46 \pm 1.04(\mathrm{~N}=97)$ & $0.68 \pm 4.98(\mathrm{~N}=373)$ & 0.390 \\
\hline D-dimer & $3233.48(\mathrm{~N}=63)$ & $2860.96 \pm 4315.27(\mathrm{~N}=26)$ & $3495.25 \pm 5807.46(\mathrm{~N}=37)$ & 0.115 \\
\hline NLR (Neutrophil to Lymphocyte Ratio) & $5.11(\mathrm{~N}=886)$ & $7.71 \pm 5.76(\mathrm{~N}=129)$ & $4.66 \pm 4.33(\mathrm{~N}=757)$ & 0.000 \\
\hline PLR (Platelet to Lymphocyte Ratio) & $13.03(\mathrm{~N}=900)$ & $19.13 \pm 19.22(\mathrm{~N}=131)$ & $11.99 \pm 15.59(\mathrm{~N}=769)$ & 0.001 \\
\hline
\end{tabular}

Table 4: Expired/Alive.

\begin{tabular}{|c|c|c|c|c|}
\hline & Total Mean & Expired Group (Mean \pm SD) & Alive Group (Mean \pm SD) & P-Value \\
\hline CRP & $35.73(\mathrm{~N}=820)$ & $60.95 \pm 50.48(\mathrm{~N}=116)$ & $31.54 \pm 32.11(\mathrm{~N}=704)$ & 0.000 \\
\hline Troponin & $0.63(\mathrm{~N}=470)$ & $2.15 \pm 9.56(\mathrm{~N}=98)$ & $0.23 \pm 0.70(\mathrm{~N}=372)$ & 0.000 \\
\hline D-dimer & $3233.48(\mathrm{~N}=63)$ & $3998.40 \pm 5055.44(\mathrm{~N}=25)$ & $2730.25 \pm 5322.59(\mathrm{~N}=38)$ & 0.631 \\
\hline NLR (Neutrophil to Lymphocyte Ratio) & $5.11(\mathrm{~N}=886)$ & $8.98 \pm 5.84(\mathrm{~N}=116)$ & $4.52 \pm 4.19(\mathrm{~N}=770)$ & 0.000 \\
\hline PLR (Platelet to Lymphocyte Ratio) & $13.03(\mathrm{~N}=900)$ & $24.50 \pm 30.57(\mathrm{~N}=119)$ & $11.29 \pm 11.99(\mathrm{~N}=781)$ & 0.000 \\
\hline
\end{tabular}

Table 5: Logistic regression.

\begin{tabular}{|c|c|c|c|c|c|c|c|c|c|}
\hline & \multicolumn{3}{|c|}{ Severity } & \multicolumn{3}{|c|}{ ICU } & \multicolumn{3}{|c|}{ Expired } \\
\hline & OR & $95 \% \mathrm{Cl}$ & P-value & OR & $95 \% \mathrm{Cl}$ & P-value & OR & $95 \% \mathrm{Cl}$ & P-value \\
\hline NLR & 0.090 & $1.060-1.130$ & 0.000 & 0.106 & $1.074-1.151$ & 0.000 & 0.152 & $1.121-1.228$ & 0.000 \\
\hline PLR & 0.011 & $1.002-1.020$ & 0.016 & 0.020 & $1.009-1.031$ & 0.000 & 0.043 & $1.030-1.058$ & 0.000 \\
\hline CRP & 0.016 & $1.012-1.020$ & 0.000 & 0.014 & $1.009-1.018$ & 0.000 & 0.017 & $1.012-1.022$ & 0.000 \\
\hline Troponin & 0.560 & $1.181-2.594$ & 0.005 & -0.15 & $0.921-1.055$ & 0.669 & 0.358 & $1.098-1.861$ & 0.008 \\
\hline D-dimer & 0.000 & $1.000-1.000$ & 0.011 & 0.000 & $1.000-1.000$ & 0.633 & 0.000 & $1.000-1.000$ & 0.346 \\
\hline
\end{tabular}

Table 6: ROC.

\begin{tabular}{|c|c|c|c|c|c|c|c|c|c|c|c|c|c|c|c|}
\hline & \multicolumn{5}{|c|}{ Severity } & \multicolumn{5}{|c|}{ ICU } & \multicolumn{5}{|c|}{ Expired } \\
\hline & AUC & OT & $\begin{array}{c}\text { Sensitivity } \\
(\%)\end{array}$ & $\begin{array}{c}\text { Severity } \\
(\%)\end{array}$ & P-value & AUC & OT & $\begin{array}{c}\text { Sensitivity } \\
(\%)\end{array}$ & $\begin{array}{c}\text { Severity } \\
(\%)\end{array}$ & P-value & AUC & OT & $\begin{array}{c}\text { Sensitivity } \\
(\%)\end{array}$ & $\begin{array}{c}\text { Severity } \\
(\%)\end{array}$ & P-value \\
\hline NLR & 0.688 & $\geq 4.61$ & 58.9 & 70 & 0.000 & 0.701 & $\geq 5.29$ & 56.6 & 79.9 & 0.000 & 0.787 & $\geq 6.03$ & 62.1 & 80 & 0.000 \\
\hline PLR & 0.629 & $\geq 10.92$ & 52.2 & 79.3 & 0.000 & 0.673 & $\geq 11.70$ & 55.7 & 70 & 0.000 & 0.733 & $\geq 14.71$ & 53.8 & 80 & 0.000 \\
\hline CRP & 0.672 & $\geq 33.25$ & 56.2 & 70 & 0.000 & 0.625 & $\geq 42.95$ & 50 & 74.6 & 0.000 & 0.67 & $\geq 51.8$ & 50 & 81 & 0.000 \\
\hline Troponin & 0.629 & $\geq 0.162$ & 50.6 & 73.4 & 0.000 & & & & & & 0.654 & $\geq 0.187$ & 55.1 & 71 & 0.000 \\
\hline D-dimer & 0.712 & $\geq 829.0$ & 65.5 & 73.5 & 0.004 & & & & & & & & & & \\
\hline
\end{tabular}

study, except D-dimer, were an independent risk factor for the death.

ROC curves were drawn for each independent risk factor separately in each category based on the results of the binary logistic regression analysis. AUC, optimal threshold, and p-values are shown in Table 6.

\section{Discussion}

As we have mentioned previously at the end of the year 2019, a pandemic disease that was called Covid-19 by WHO affected human's life [18-20]. This newfound disease had a wide range of signs and symptoms with no steady pattern within different groups of people. Clinical presentations were different from no symptoms to multiple organ failure and death [4]. As the disease had no predictable clinical pattern and may cause different clinical presentations in different people, finding some predictive factors can help in managing the disease and doing early invasive procedures if needed that leads to lower mortality rate. Different studies in the mechanism of the disease have shown the important role of different inflammatory factors. So, finding statistically significant values in inflammatory factors can help us in managing the disease. In this study we focused on CRP, NLR, PLR, D-dimer and Troponin as predictive factors in three different categories of patients: a) Severe/Moderate; b) ICU add/Non-ICU add, and c) Expired/Alive.

\section{Severe/Moderate}

Out of 1199 patients who were admitted to our hospitals 908 were in the severe group. All of the factors were higher in the severe group and this difference was statistically significant (Table 2). Binary logistic regression was done for parameters with $\mathrm{P}$-value $<0.05$ separately and it showed that all of them can predict the severity of the disease (Table 5). ROC curve was drawn for all the factors separately based on the binary logistic regression analysis (Table 6 and Figure1). Our study and the obtained results suggested that all of these factors can play a role in predicting the severity of the disease as an independent risk factor. The ROC curve suggests the optimal threshold of $\geq 4.61$ for NLR, $\geq 10.92$ for PLR, $\geq 33.25$ for CRP, $\geq 0.162$ for Troponin, and $\geq 829.00$ for $\mathrm{D}$-dimer which are all statistically significant with 

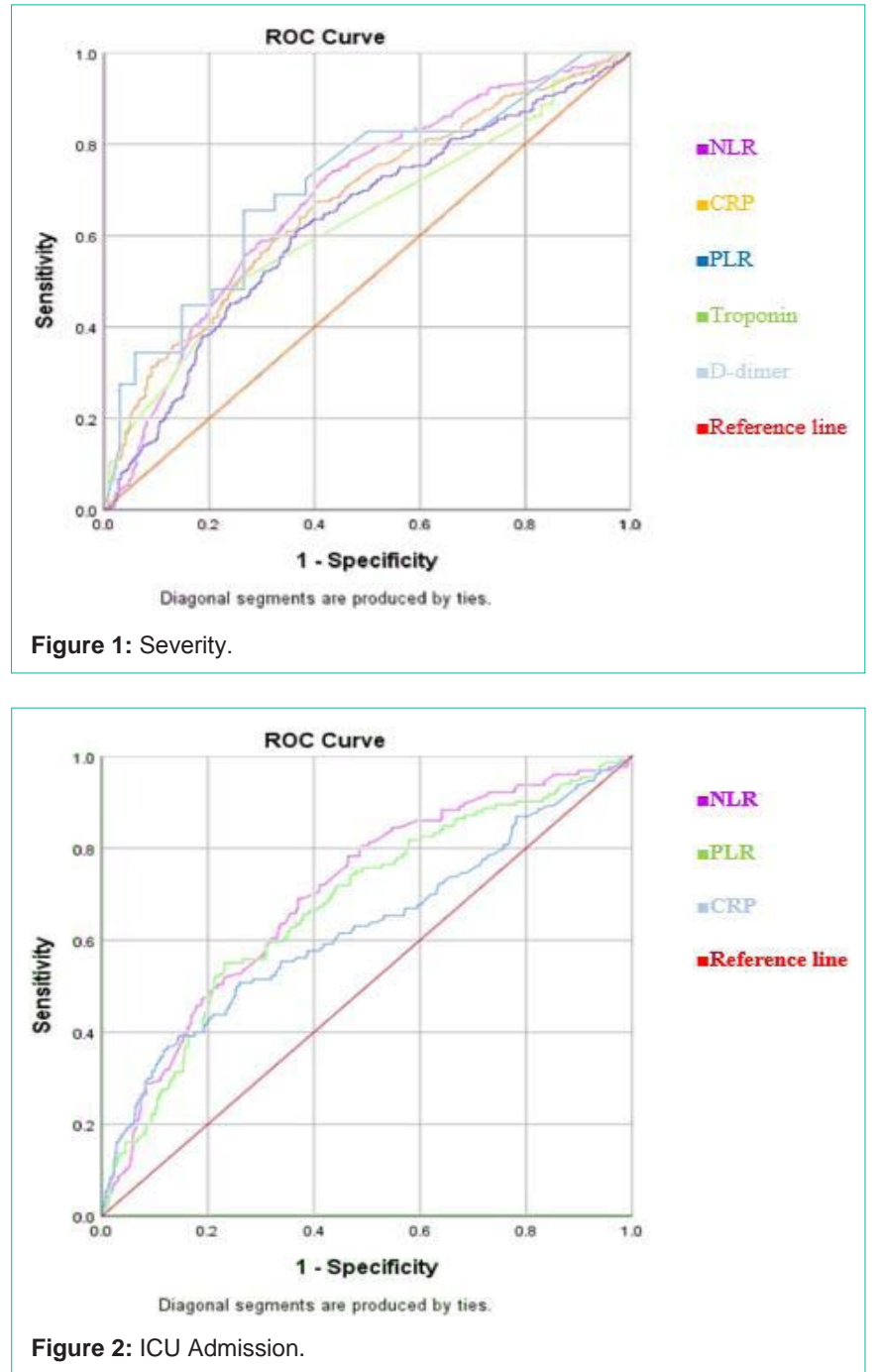

$\mathrm{P}$-value $<0.05$. Therefore, it seems that detecting these values on the first day of admission can be useful in predicting the severity of the disease and the condition of the patient (besides the clinical factors that are currently being used). One systematic review which was done in Wuhan, China on 29 articles has also shown the prognostic value of CRP and NLR [21]. Some other studies which were done on the changes of the blood cell changes during the disease have shown the thrombocytopenia and the leukopenia in the severe group of patients that results in the higher levels of the PLR in this group of patients [22]. Also some other researches have been done on the changes of the level of Troponin in patients with the Covid-19 that have shown the statistically significant rise in the level of Troponin but the main mechanism still remains unknown [23].

\section{ICU add/Non-ICU add}

143 out of 1199 patients were admitted to the Intensive Care Unit (ICU) on their arrival. All the factors that have been investigated in this study were higher in the ICU add group and this difference was statically significant for NLR (P-value: 0.000), PLR (P-value: 0.001), and CRP (P-value: 0.000) (Table 3). Binary logistic regression was done for the factors with $\mathrm{P}$-value $<0.005$ (Table 5).

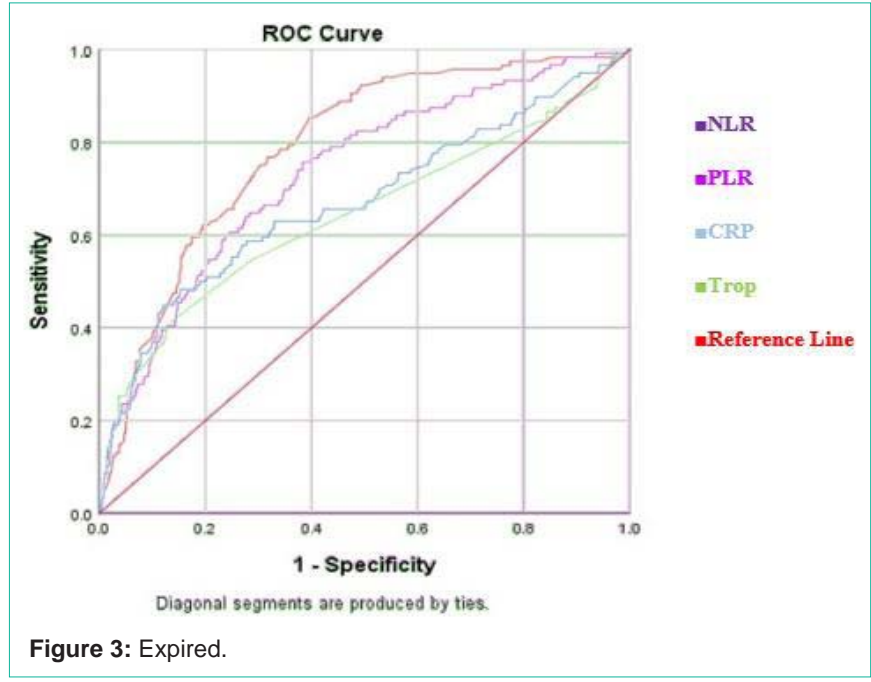

The ROC curve was drawn based on the results of the binary logistic regression (Table 6 and Figure 2). The study shows that the inflammatory factors NLR, PLR, and CRP can independently predict the need of ICU admission in the Covid-19 patient, so detecting these factors at the first date of the admission can be helpful. The ROC curve suggests the optimal threshold of $\geq 5.29$ for NLR, $\geq 11.70$ for PLR, and $\geq 42.95$ for CRP. Although the increase in D-dimer in the ICU add group was not statistically significant, other studies have also shown the increase in D-dimer level in this group of patients and their tendency to DIC (Disseminated intravascular coagulation) [24].

\section{Expired/Alive}

144 patients of all, expired at the first date of their admission. We studied all the factors between these two groups. The results showed that all the factors were higher in the expired group and were all statically significant $(\mathrm{P}$-value $<0.005)$ except the $\mathrm{D}$-dimer $(\mathrm{P}$-value: 0.631 ) (Table 4 and Figure 3). Binary logistic regression was done for factors with P-value $<0.005$ and the ROC curve was drawn based on the results of the logistic regression (Table 5 and 6). Our Study shows that all of these factors can be used for the prediction of the mortality of the disease. The suggested optimal thresholds based on ROC curve are $\geq 6.03$ for NLR, $\geq 14.71$ for PLR, $\geq 51.8$ for CRP, and $\geq 0.187$ for Troponin.

\section{Conclusion}

In conclusion our study shows that beside factors like age, past medical or drug history (e.g. respiratory diseases or consumption of corticosteroids) or the level of blood oxygen [19], the changes of the inflammatory factors in the laboratory findings on the first date of the admission can be used to predict the process of the disease which helps the system to control the burden of the disease and may cause a reduction in the mortality rate of the Covid-19.

\section{Limitations}

Our study has some priorities in comparison to previous ones and has met some of their limitations. It was a multicenter study and included the patients of three different hospitals $[9,25]$. The size of the population that we studied, was much bigger than the previous ones so the results of our statistical analyses are more reliable $[2,25]$. 
We excluded patients with comorbidities that could affect the results of our study about inflammatory factors (e.g. autoimmune diseases) [7], and the study was done within three different groups and it was not only about the severity. In spite of the above-mentioned strengths points of this study there were three main limitations for it. First, it was a retrospective study. The next one is that the factors are not measured in all of the patients and each factor has its sample which differs from the others making it impossible to study the correlation between the factors and each one must be studied as an independent factor. The third and the last one is that we didn't study the role of the cytokines like IL6 that have been pointed out in many other studies.

\section{References}

1. Johns Hopkins University \& Medicine. COVID-19 map. Baltimore, MD: Johns Hopkins University. 2020.

2. Liu Y, Du X, Chen J, et al. Neutrophil-to-lymphocyte ratio as an independent risk factor for mortality in hospitalized patients with COVID-19. J Infect. 2020 81: e6-e12.

3. Fu J, Kong J, Wang W, et al. The clinical implication of dynamic neutrophil to lymphocyte ratio and D-dimer in COVID-19: A retrospective study in Suzhou China. Thromb Res. 2020; 192: 3-8.

4. Li Q, Guan X, Wu P, et al. Early Transmission Dynamics in Wuhan, China of Novel Coronavirus-Infected Pneumonia. N Engl J Med. 2020; 382: 11991207.

5. Pedersen SF, Ho YC. SARS-CoV-2: a storm is raging. J Clin Invest. 2020 130: 2202-2205

6. Wu Z, McGoogan JM. Characteristics of and Important Lessons from the Coronavirus Disease 2019 (COVID-19) Outbreak in China: Summary of a Report of 72,314 Cases from the Chinese Center for Disease Control and Prevention. JAMA. 2020; 323: 1239-1242.

7. Cheng B, Hu J, Zuo X, et al. Predictors of progression from moderate to severe coronavirus disease 2019: a retrospective cohort. Clin Microbiol Infect. 2020; 26: 1400-1405.

8. N Zhu, D Zhang, W Wang, X Li, B Yang, J Song, et al. A Novel Coronavirus from Patients with Pneumonia in China, 2019. N. Engl. J. Med. 2020; 382 727-733.

9. Yang AP, Liu JP, Tao WQ, Li HM. The diagnostic and predictive role of NLR, d-NLR and PLR in COVID-19 patients. Int Immunopharmacol. 2020; 84: 106504.

10. Qin C, Zhou L, Hu Z, et al. Dysregulation of immune response in patients with COVID-19 in Wuhan, China. Clin Infect Dis. 2020; 71: 762-768.
11. Fu J, Kong J, Wang W, et al. The clinical implication of dynamic neutrophil to lymphocyte ratio and D-dimer in COVID-19: A retrospective study in Suzhou China. Thromb Res. 2020; 192: 3-8.

12. Tersalvi G, Vicenzi M, Calabretta D, Biasco L, Pedrazzini G, Winterton D. Elevated Troponin in Patients With Coronavirus Disease 2019: Possible Mechanisms. J Card Fail. 2020; 26: 470-475.

13. CD Russell, A Parajuli, HJ Gale, NS Bulteel, P Schuetz, C de Jager, et al The utility of peripheral blood leucocyte ratios as biomarkers in infectious diseases: a systematic review and meta-analysis. J Infect. 2019; 78: 339-348.

14. E Huguet, $G$ Maccallini, $P$ Pardini, $M$ Hidalgo, S Obregon, $F$ Botto, et al. Kotliar, Reference values for neutrophil to lymphocyte ratio (NLR), a biomarker of cardiovascular risk, according to age and sex in a latin american population. Curr Probl Cardiol. 2021; 46: 100422.

15. JS Park, KW Seo, BJ Choi, SY Choi, MH Yoon, GS Hwang, et al. Importance of prognostic value of neutrophil to lymphocyte ratio in patients with STelevation myocardial infarction. Medicine (Baltimore). 20108; 97: e13471.

16. Z Mei, L Shi, B Wang, J Yang, Z Xiao, P Du, et al. Prognostic role of pretreatment blood neutrophil-to-lymphocyte ratio in advanced cancer survivors: a systematic review and meta-analysis of 66 cohort studies. Cancer Treat Rev. 20017; 58: 1-13.

17. Chan AS, Rout A. Use of Neutrophil-to-Lymphocyte and Platelet-toLymphocyte Ratios in COVID-19. J Clin Med Res. 2020; 12: 448-453.

18. Kuppalli K, Rasmussen AL. A glimpse into the eye of the COVID-19 cytokine storm. E Bio Medicine. 2020; 55: 102789.

19. World Health Organization.

20. Iran's National Guideline for 2019-nCoV. $2^{\text {nd }}$ edition.

21. Feng X, Li S, Sun Q, Zhu J, Chen B, Xiong M, et al. Immune-Inflammatory Parameters in COVID-19 Cases: A Systematic Review and Meta-Analysis. Front Med (Lausanne). 2020; 7: 301.

22. Qu R, Ling Y, Zhang YH, Wei LY, Chen X, Li XM, et al. Platelet-to-lymphocyte ratio is associated with prognosis in patients with coronavirus disease-19. J Med Virol. 2020; 92: 1533-1541.

23. Tersalvi G, Vicenzi M, Calabretta D, Biasco L, Pedrazzini G, Winterton D. Elevated Troponin in Patients With Coronavirus Disease 2019: Possible Mechanisms. J Card Fail. 2020; 26: 470-475.

24. Liu F, Li L, Xu M, Wu J, Luo D, Zhu Y, et al. Prognostic value of interleukin-6, $\mathrm{C}$-reactive protein, and procalcitonin in patients with COVID-19. J Clin Virol. 2020; 127: 104370.

25. Shang W, Dong J, Ren Y, Tian M, Li W, Hu J, et al. The value of clinical parameters in predicting the severity of COVID-19. J Med Virol. 2020. 